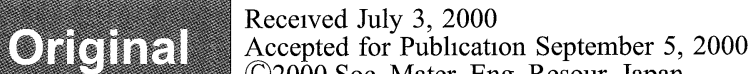

(C)2000 Soc. Mater. Eng. Resour. Japan

\title{
DIRECT COMPARISONS BETWEEN VIRTUAL AND EXPERIMENTAL POWDER MIXTURES
}

\author{
R.E. Riman*, D-G Han*, and E.A. Gulliver* \\ *V.A. Greenhut, Rutgers University, Department of Ceramic and Materials Engineering, Piscataway, NJ, \\ J. Cesarano, Sandia National Laboratories, Albuquerque, NM, P. Hoeper, UPS, Somerset, NJ, \\ T. Stark, Materials Analytical Services, Raleigh, NC, J. Friel, Princeton Gamma Tech, Princeton, NJ. \\ E-mail :RRiman@aol.com
}

\begin{abstract}
The objective of this research is to develop criteria for improving mixedness by proper selection of the powder components. Our two-pronged approach involves both computation and experimental determination of mixedness. Computation of mixedness utilizes a modular program that incorporates powder components and packs them via dropping and rolling. The virtual mixture is interrogated using concentric shell and tessellation analysis. The experimental determination of mixedness begins with epoxy-embedded microstructures. A focused ion beam produces smooth planar cross-sections for mixture analysis of a wide range of materials. Tessellation analysis of high resolution x-ray maps is used to interrogate the mixture, offering us the ability to compute tile area distributions, which allows us to compare the structure of simulated and experimental systems. Having this capability allows us to develop a benchmark for the mixedness possible in a chosen system. In addition, it allows us to develop techniques for improving that benchmark through variation of the powder component characteristics.
\end{abstract}

Key Words : Ceramics, mixing processes, determination of mixedness, distribution of powder components, chemical homogeneity

\section{Introduction}

Powder mixedness pertains to spatial and intensity fluctuations of discreet chemical components in a bed of defined porosity and packing hierarchy. Each component corresponds to powders of a specified size and size distribution with spherical morphology. Mixedness pertains to a particulate structure that is either fully randomized, a partially ordered intermediate state, such as particles with a surface coating, or a crystalline structure such as the colloidal crystal systems now being examined for photonic band-gap materials. Mixture quality is a specific aspect of mixedness. It is the variance, or intensity, of compositional fluctuations at a length scale critical to the mixture. When one engineers degree of mixedness, there are two choices in controlling the process. First, one can choose to control the structure of the mixture or the mixedness by optimizing the unit operations in the process. In fact, this is the most common way to control mixedness. However, there is another important step in controlling the mixedness of a powder bed, and that is to examine the characteristics of the starting powder. In particular, we may be referring to the characteristics of particle size, the particle size distribution, or it could be the incorporation of a specific precursor for a cation component. For instance, a high molecular weight of an "A" cation component could be advantageous in improving the mixedness of a microstructure over what could be achieved with a low molecular weight precursor.

When looking at the mixedness of a structure, two parameters are important. One is the scale of segregation, where we study how characteristics such as compositional fluctuation changes as the particle sizes vary. For example, as particle size is reduced, the scale of segregation decreases. Another is the scale of intensity, where the magnitude of the concentration gradient as we traverse from particle to particle varies with component choice. Mixing intensity can be changed by component choice. Mixing two components that contain at least one common cation (e.g., $\mathrm{MgO}$ and $\mathrm{MgAl}_{2} \mathrm{O}_{4}$ ) results in a lower mixing intensity scale than choosing two particles that do not contain a common cation (e.g., $\mathrm{MgO}$ and $\left.\mathrm{Al}_{2} \mathrm{O}_{3}\right)$.

Controlling mixedness is important for many reasons. For traditional powder processing, improved mixedness accelerates the reaction kinetics and leads to phase-pure products, owing to the decrease in diffusion distance. In addition, many ceramic processes incorporate sintering additives. While each additive must be incorporated uniformly on a spatial scale, their low concentration presents a formidable challenge toward achieving a targeted mixedness. All of the issues are pertinent, not only to powder synthesis as mentioned above, but also to powder processing as a tool for generating controlled microstructures. The distribution of components should not be limited to ceramic components, but should also include organic components such as, dispersants, binders and plasticizers. All of the components in a mixture function in a much more potent fashion when their distribution is optimized.

The most visible example of the advantages of distributing a component in a uniform fashion can be seen when looking at a 
two-component mixture (Figure 1). In a fully-random structure, containing particles A and B, the location and the identify of these particles are in fact predicted by random probability alone. We can compare this to a structure that is partially ordered, such as large particles called "A" coated by finer particles called "B". If A and $B$ react to give " $\mathrm{C}$ ", the product $\mathrm{C}$ will form at the interface between $\mathrm{A}$ and $\mathrm{B}$. In a random structure, the distance between the interfaces producing $\mathrm{C}$ will be randomly distributed, meaning that there will be a broad range of diffusional distances. In the case of coated particles, the distance between interfaces producing $\mathrm{C}$ is far smaller. Now, in reality, most systems are not based on two-component mixtures. Many more than two components are typically used in a ceramic system and thus, the processing of random mixtures must be employed, instead. In addition, most unit operations are highly capable of producing random structures but less capable of producing completely ordered structures. The critical question is, what is the average and the distribution of diffusional distances existing in the powder bed (compact), and how can controlling the structure of a mixture be used to minimize these distances? While looking at this particular example, one can see the advantages of being able to engineer the spatial and intensity scale of mixedness to the particular specifications set by the product properties. Thus, we will focus on how we choose to optimize mixedness of a randomly mixed powder bed of particulate components that can number two or more.

Examining 40 years of statistical approaches towards mixedness problems, various analytical expressions have been derived to explain how the variance of a mixture is influenced by changes in particle size. However, there are serious limitations to this approach. One of the most serious limitations is that most of the statistical approaches that have been developed, have centered on getting a representative sample of a population. This approach requires sampling a large number of particles (e.g., a large scoop

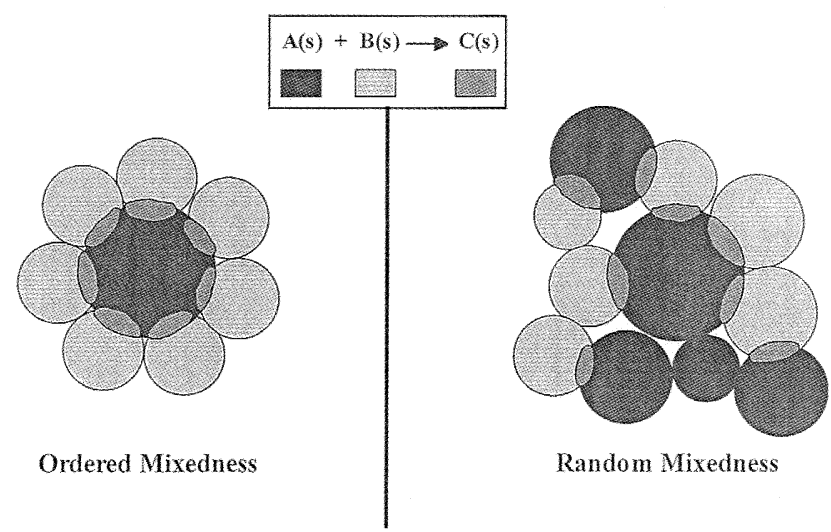

Figure 1. This is a comparison of the impact of ordered vs. random mixedness on a two component solid-state reaction (e.g. A reacts with $B$ to form $C$ ). In ordered mixedness the contact between $\mathrm{A}$ and $\mathrm{B}$ is optimized and the formation of the product $\mathrm{C}$ is not limited by the availability of $\mathrm{B}$ to $\mathrm{A}$. In a system having random mixedness, the contact between $\mathrm{A}$ and $\mathrm{B}$ is not optimized and the formation of $\mathrm{C}$ is limited by the availability of B to A. For example, in the case of random mixedness, one of the particles of $\mathrm{A}$ is not in contact with any of the particles of $\mathrm{B}$ and therefore cannot immediately participate in the reaction to form $\mathrm{C}$. of the mixture). However, for ceramic processing, it is nearest neighbor interactions (micromixing) that count the most. Taking a relatively large scoop out of a mixture, which is what statistical analyses focus upon, does not reveal the nearest neighbor interactions. Instead, they reveal a much larger collective interaction of particles or in other words, the macromixing scale. In order to focus on getting these nearest neighbor interactions, a different approach needs to be taken, one approach is the concentric shell model of mixedness (CSMM). For the CSMM, we take a given microstructure, which is a randomly packed ensemble of components of varying size, and we simply take concentric shell slices through the mixture (Figure 2a). Within each of these concentric shells, we add up the contributions for each of the component particles that happen to fall within the boundaries of the shell and sum the component contributions cumulatively over a radial distance. This numerical approach toward analyzing a mixture is central to how we approach the optimization of mixedness.

This paper is divided in two different sections: (1) how mixedness is computed and (2) how to experimentally determine mixedness. Ultimately we not only want to devise engineering strategies for optimizing the mixedness of a structure, but we also want to be able to measure it in real systems and validate our modeling approach.

\section{COMPUTATIONAL DETERMINATION OF MIXEDNESS}

In our computational work, the main goal is to develop user-
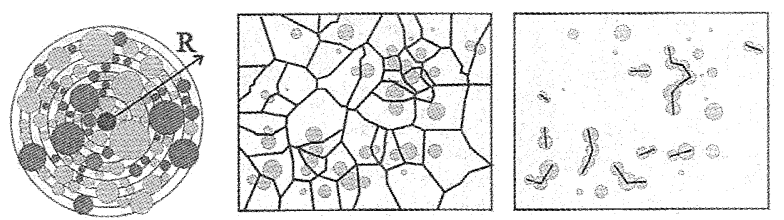

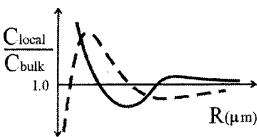

(a) CSMM or RDF

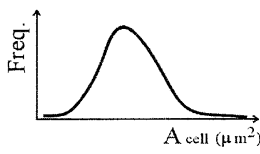

(b) Tessellation

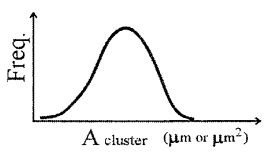

(c) Percolation Cluster
Figure 2. Mixedness analysis methods. a) The concentric-shell model of mixedness (CSMM) starts from a point in the mixture and calculates the ratio of components contained in a series of concentric spheres. A radial distribution function (RDF) operates in the same way except that it only counts the number of particle centers contained within each of the concentric spheres. In both CSMM and RDF the measurements from many initial starting points are collected and plotted as a function of sphere radius. b) Tessellation analysis isolates the particles of a single component. Boundaries are then drawn about the particles in such a way that all of the material within the boundary is closer to the particle at its center than it is to any other particle in the mixture. The frequency of tiles as a function of tile size or composition can then be used to characterize the mixture. Tessellation can be applied in both $2 \mathrm{D}$ and $3 \mathrm{D}$ but 2D tessellation allows direct comparison with cross sections of green bodies. c) Percolation cluster analysis examines the clusters or networks formed by particles of the same component in contact with each other. The frequency of clusters as a function of size or density can then be used to characterize the mixture. 
friendly mixedness simulation software that can generate output parameters that are directly comparable to experimental mixedness data. There are several reasons for developing this software. One is that we would like to establish a mixture quality assessment protocol. That is, we would like to develop a methodology for which this software can be used for approaching a problem. We would also like to have software that will provide both the ceramic processing and the general powder processing community with a simulation tool for a variety of engineering problems. Potential applications include developing specifications for starting powders that can be used for distinguishing good candidate powders from poor ones. In addition, we would like to be able to use this software to provide a benchmark. Currently there is no benchmark for mixture quality in industry. Thus, the ceramic community does not have an awareness of whether or not the mixing methods we are currently using mix on a level that would approach an ideal random mixture of particles. Most of our assessments are currently done based on a non-quantitative visual (microscopy) or indirect (sintering behavior, electric properties) basis, which at best provides a qualitative assessment of the mixture quality.

The first step in developing a model for mixedness analysis is to first be able to create a virtual green microstructure. In virtual space, we need to fabricate a green microstructure of particles whose positions and identity (size, composition) are determined by random probability alone. For monodisperse systems, there are simple statistical methods to build multi-component mixtures. However, if one incorporates polydispersed multicomponent systems that are reflective of what are actually used in industry, producing such a structure is no longer a trivial matter, primarily because of the problem of particle overlap with unreasonably large void spaces. We have examined several packing models which include the concentric shell model [1], a dropping and rolling model [2] and an expanding lattice model [3]. Our work to date has focused mostly on a dropping and rolling model developed at Sandia National Labs. This model is based on achieving minimum potential energy by the dropping and rolling of particles, which are added one by one to a container that holds the particles.

Unfortunately, the dropping and rolling model has no protocol for checking whether this packing model produced any type of non-random artifacts. One of the ways to examine this model more closely is to produce cross-sections of the mixture where we can look at the positions and areal number densities for each size-class of particles. If desired we can also look at the full distribution of sizes. We have examined model mixtures to test this approach. For instance, if you look at particles that differ in size by an order of magnitude (e.g., $1 \mu \mathrm{m}$ and $10 \mu \mathrm{m}$ ) the dropping and rolling model can actually produce a very highly skewed distribution of particles. Most of the fine particles end up at the bottom of the container, having percolated through the interstices of all the larger particles. As you increase the size of the smaller particles, this segregation effect decreases. Once you get to mixtures where the particle sizes are equal the skew is eliminated with the exception of the particles whose positions are governed by their proximity to the wall. While this type of segregation can be a very troubling issue, we are currently working on a technique for studying how these distributions can be randomized by adjusting a parameter called the sticking factor. The sticking factor prevents the particles from simply rolling and dropping through the interstices of the packed particle structure by reducing their mobility. We are now developing a computer-automated approach for adjusting the
Table 1. Determination of the characteristic length scale for Co in a WC$\mathrm{Co}-\mathrm{VC}$ particle mixture as a function of the Co powders obtained from two different suppliers (Eurotungstene and H.C. Stark).

\begin{tabular}{lcc}
\hline & $\begin{array}{c}\text { Eurotungstene } \\
\text { Cobalt }\end{array}$ & $\begin{array}{c}\text { H. C. Starck } \\
\text { Cobalt }\end{array}$ \\
\hline Mean Concentration Length Scale $(\mu \mathrm{m})^{\dagger}$ & 3.38 & 4.45 \\
Compositional Variance Length Scale $(\mu \mathrm{m})^{\ddagger}$ & 3.45 & $>8$ \\
Characteristic Length Scale of the Mixture $(\mu \mathrm{m})^{\S}$ & 3.45 & $>8$ \\
\hline
\end{tabular}

${ }^{\dagger}$ The length scale for mean Co concentration was chosen to be the point on the concentration vs. length scale curve where the mean Co concentration first comes within $1 \%$ of the Co concentration in the bulk mixture.

${ }^{\ddagger}$ The length scale for the variance of the Co concentration was chosen to be the point where the variance of the Co concentration dropped below $0.001{ }^{\S}$ The characteristic length scale for Co in each mixture was then taken to be the longer of the length scales obtained from the mean and variance of the Co concentrations.

sticking factors that should help us build random multicomponent green microstructures in virtual space.

If one assumes the mixedness structures that our model creates are random, the next step is to interrogate the structure with structure-determining tools. First, we need to produce a section of the mixture. To conserve on computational power and enable the program to run on a personal computer, we have focused on timeeffective ways of acquiring and controlling the database of the particle positions in the compact. This database enables us to access any arbitrary section of the structure. The sectioning technique is very important because it allows us assess the attributes of the mixture at random locations. It also allows us to deliberately choose specific orientations of mixture. This is critical for studying non-random microstructures such as colloidal crystals. Given a section of the green body, we can do four different things with it (Figure 2). First, we can analyze it on a two-dimensional basis using tessellation analysis, where we draw cells whose walls bisect between neighboring particles of like components. Second, we can look at connectivity in the mixture by analyzing the percolation length, which is the distribution of length scales in which a specific component neighbors another particle of the same component. Third, we can construct a radial distribution function between pairs of particles of like components. Fourth, we can use a 3-D concentric shell model, which we mentioned earlier. We start with a reference particle, which can be either of a deliberate chosen component and size or a randomly one. Concentric shells are then drawn or sliced through the green compact mixture in order to assess the distribution of the components in a cumulative radial fashion. Each of these different approaches has a different utility for analyzing a mixedness structure. In this paper, we will only cover tessellation and concentric shell analysis.

Concentric shell analysis is quite effective for computing characteristic length scales. This analysis is performed in as many as 1000 regions of a mixture, enabling the computation of a mean concentration and variance as a function of length scale. Tolerance limits are set that are acceptable for the materials application of interest. These tolerance limits should be set in terms of the spatial mean concentration and spatial variance. By calculating these parameters, we now have criteria to compare different sources of 
powders. Quite frequently, we desire to pick the component that minimizes the length scale for a specific mean concentration and compositional variance. For example, in Table 1 we compare the influence of cobalt $(\mathrm{Co})$ powders obtained from two different suppliers on the characteristic length scale of Co in a mixture of tungsten carbide (WC), Co and vanadium carbide (VC) powders. In this case, the WC and VC powders were the same for both mixtures. In this particular case, the $\mathrm{Co}$ powder from Eurotungstene provides a far smaller length scale for the mean Co concentration than the Co powder obtained from H.C. Stark. In addition, the length scale for the concentration variance of the Eurotungstene Co powder is also a far smaller than that obtained from the HC-Stark Co powder. In this particular case, we have defined the characteristic length scale for a WC-Co-VC mixture to be the longer of the length scales obtained from the mean Co concentration and the variance of the Co concentration. In addition, we have established that Co powder obtained from Eurotungstene will produce a shorter characteristic length scale than the Co powder obtained from H.C. Starck.

\section{EXPERIMENTAL DETERIMINATION OF MIXEDNESS}

Our technical goals are as follows: (1) develop a protocol for processing a green (unfired) microstructure that can be interrogated without modifying any of the positions of the various particles in the bed and (2) have an analysis method that could be directly comparable to the types of analysis output obtained from our mixedness software. The starting point is to be able to take a given green microstructure and section it. If conventional methodologies are used for sectioning, serious problems prevail. When one produces a polished section, a common problem is the presence of pullouts. If one chooses instead to use ultramicrotomy, the number of pullouts is vastly reduced. However, ultra-microtomy is a tedious process that requires a great deal of experience and time. In response to these issues, a new approach was developed where a focused ion beam is used to section through the mixture. A focused ion beam can generate a very smooth surface and cut through submicron (Figure 3). In addition, we can also section through very hard materials such as WC-Co-VC mixtures and again, we can easily see submicron features. Fine scale images such as these allow us to use image analysis procedures to do the same types of analyses that were discussed before with the mixedness software, such as tessellation analysis, radial distribution functions or percolation length. When there is very little contrast between components, we can use energy dispersive spectroscopy to map the particle chemistry. Advances in field emission scanning electron microscopy (FE-SEM) enable us to interrogate samples using very low accelerating voltages and generate energy dispersive spectroscopy maps of component chemistry at very high resolution $(\sim 50 \mathrm{~nm})$. This advance facilitates the examination of mixture microstructures on a sub-micron scale. Figure 4 shows a cobalt-tungsten carbide mixture that was sectioned by focused ion beam milling, interrogated with energy dispersive spectroscopy, converted to a binary image and then tessellated.

If one takes this tessellation and analyzes it, direct comparison with a tessellation generated by our mixedness software is possible. The tessellation structure generated by the mixedness software is effectively a benchmark structure. It defines the optimum state of mixedness based on the initial starting powder characteristics. The experimental tessellation structure describes what our process is able to achieve. Tile area distributions can be calculated from each image and compared to determine whether the mixedness achieved was similar to an ideal random mixture of particles. When these two distributions are compared for this specific example, the surprising result is that the experimental system has a far smaller tessellation tile area distribution. The reason for this is probably because during the course of mixing, this system is likely to have undergone a particle size reduction (carbides are typically mixed and comminuted in an attrition mill). So, in order to validate our computational model, we would have to know what the size distribution of the particle components after milling was performed.

In order to better validate our model, we are doing research with paradigm particle systems, which do not require comminutionbased approaches for mixing. Thus, our ideal systems incorporate mixtures of narrow size distribution aluminum oxide and monodispersed silica. In the near future, we will be conducting work with these systems to assess whether or not we can use our mixing software as a benchmarking tool.

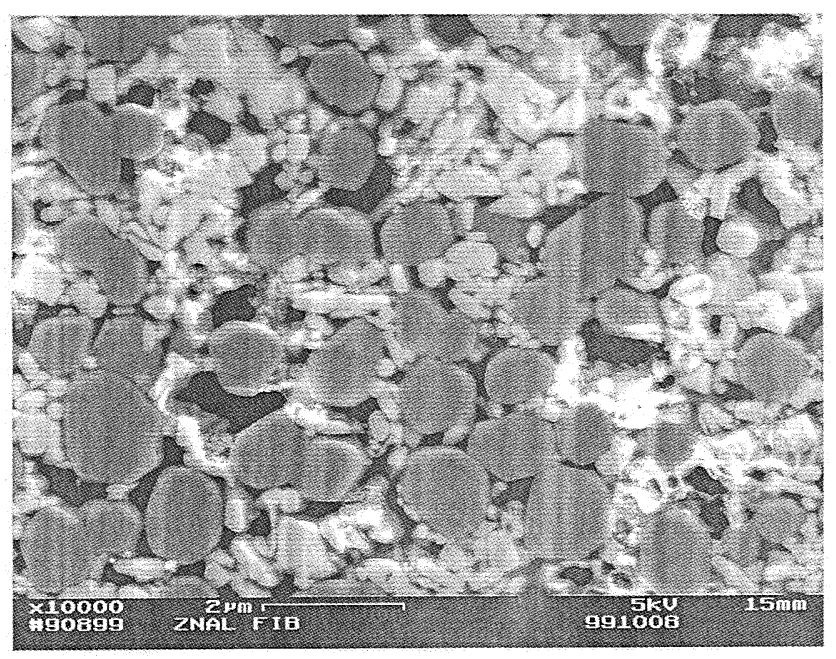

Figure 3. A cross-section of a green body consisting of $\mathrm{Al}_{2} \mathrm{O}_{3}$ and $\mathrm{ZnO}$ particles. $\mathrm{Al}_{2} \mathrm{O}_{3}$ particles are darker gray and equi-axed. $\mathrm{ZnO}$ particles are a lighter gray and have a broad variety of different shapes.
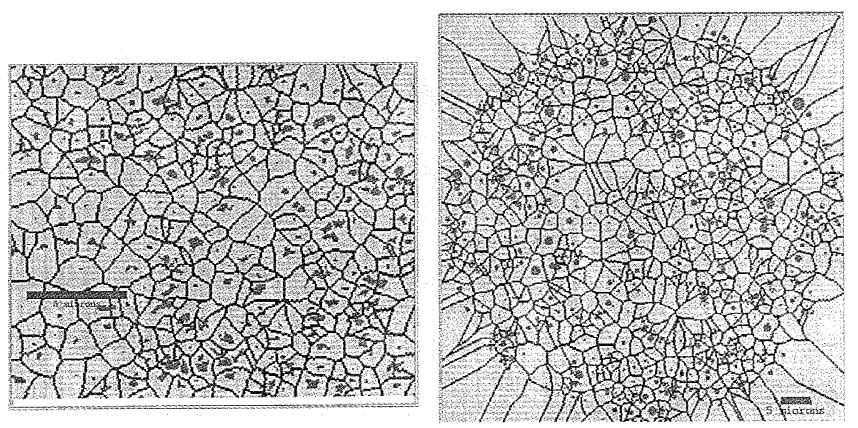

Figure 4. The tessellation on the left was obtained from an EDS map of the cobalt distribution in a cross section of tungsten carbide/cobalt cermet green body. The tessellation on the right was obtained from the simulation of the same system. The scale bars in each image are equal to 5 microns. 


\section{SUMMARY}

We have introduced the general concept of mixedness. In addition, a computational protocol was established for calculating mixedness benchmarks as well as specifying starting powders based on quantitative criteria. Furthermore, an experimental approach for determining mixedness has been developed and we have demonstrated a protocol that can be useful for comparing computational and experimental mixedness.

\section{ACKNOWLEDGEMENTS}

We would like to acknowledge support of the Center for Ceramic Research, the National Science Foundation, and the New Jersey Commission on Science and Technology. We would like to thank
Dr. Eddy Prestridge of (Princeton Gamma Tech) for his advice on image analysis.

\section{REFERENCES}

[1] P. R. Mort III and R. E. Riman, "Determination of homogeneity scale in ordered and partially ordered mixtures", Powder Technology, 82[1], 93-104 (1995).

[2] E. M. Tory, N. A. Cochrane and S. R. Waddell, "Anisotropy in Simulated Random Packing of Equal Spheres", Nature (London), 2201023 (1968).

[3] G.T. Nolan and P.E. Kavanagh, "Computer simulation of spheres with log-normal distributions", Powder Technology, 76 309-316 (1993). 